TITLE:

\title{
Critical exponents in coupled phase-oscillator models on small- world networks
}

$\operatorname{AUTHOR}(\mathrm{S})$ :

Yoneda, Ryosuke; Harada, Kenji; Yamaguchi, Yoshiyuki Y.

\section{CITATION:}

Yoneda, Ryosuke ...[et al]. Critical exponents in coupled phase-oscillator models on small-world networks. Physical Review E 2020, 102(6):

062212.

ISSUE DATE:

2020-12-14

URL:

http://hdl.handle.net/2433/259810

RIGHT:

(C2020 American Physical Society 


\title{
Critical exponents in coupled phase-oscillator models on small-world networks
}

\author{
Ryosuke Yoneda $\odot,{ }^{*}$ Kenji Harada, and Yoshiyuki Y. Yamaguchi \\ Graduate School of Informatics, Kyoto University, Kyoto 606-8501, Japan
}

(Received 9 July 2020; revised 5 November 2020; accepted 23 November 2020; published 14 December 2020)

\begin{abstract}
A coupled phase-oscillator model consists of phase oscillators, each of which has the natural frequency obeying a probability distribution and couples with other oscillators through a given periodic coupling function. This type of model is widely studied since it describes the synchronization transition, which emerges between the nonsynchronized state and partially synchronized states. The synchronization transition is characterized by several critical exponents, and we focus on the critical exponent defined by coupling strength dependence of the order parameter for revealing universality classes. In a typical interaction represented by the perfect graph, an infinite number of universality classes is yielded by dependency on the natural frequency distribution and the coupling function. Since the synchronization transition is also observed in a model on a small-world network, whose number of links is proportional to the number of oscillators, a natural question is whether the infinite number of universality classes remains in small-world networks irrespective of the order of links. Our numerical results suggest that the number of universality classes is reduced to one and the critical exponent is shared in the considered models having coupling functions up to second harmonics with unimodal and symmetric natural frequency distributions.
\end{abstract}

DOI: 10.1103/PhysRevE.102.062212

\section{INTRODUCTION}

Ever since Huygens found that two pendulum clocks hanging on a wall swung in the opposite direction from each other, many illustrations of synchronization have been established in various fields of nature, such as frog choruses [1], flashing of fireflies [2,3], metronomes [4], and circadian rhythms [5]. It is natural to try to understand synchronization theoretically, and a coupled phase-oscillator model is one successful model to describe synchronization [6]. This model consists of many coupled oscillators, and the coupling is expressed by a periodic coupling function. Each oscillator has the so-called natural frequency, randomly drawn from a natural frequency distribution. When the coupling strength $K$ increases, the oscillators exhibit the synchronization transition from the nonsynchronized state to (partially) synchronized states. The synchronization transition is continuous or discontinuous, depending on the natural frequency distribution and the coupling function [7-17].

The critical phenomena have been extensively studied in statistical mechanics. One of their remarkable features is the existence of universality classes; the systems in a universality class share the critical exponents defined around the critical point $K=K_{\mathrm{c}}$ of a continuous transition. One of the critical exponents is $\beta$, defined by $r \sim\left(K-K_{\mathrm{c}}\right)^{\beta}$, where $r$ is the order parameter. Thus, it is natural to ask the universality classes in the coupled phase-oscillator models through values of the critical exponent $\beta$.

For the all-to-all and uniform coupling, extended studies have revealed that the value of $\beta$ depends on the coupling

\footnotetext{
*yoneda@acs.i.kyoto-u.ac.jp
}

function and the natural frequency distribution [7-17]. For simplicity, we focus on coupling functions which have two harmonics at most and review values of the critical exponent $\beta$ for the following three cases: (i) the second harmonics is absent, (ii) the second harmonics has the opposite sign from the leading harmonics, and (iii) the second harmonics has the same sign as the leading harmonics. We assume that the natural frequency distribution is unimodal and symmetric and that the second-leading term of its Maclaurin expansion is of the order $2 n$, where $n \in \mathbb{N}$. A Gaussian distribution and a Lorentzian distribution have $n=1$, for instance.

In cases (i) and (ii), the model shows a continuous transition, whereas in case (iii), a discontinuous transition occurs [12], hence we cannot define the critical exponent $\beta$. In case (i), the model becomes the Kuramoto model [7], a paradigmatic coupled phase-oscillator model. Several studies have pointed out that the critical exponent $\beta=1 /(2 n)[7-10]$. This $n$ dependence is a strong feature of the Kuramoto model and gives a sharp contrast with case (ii). In case (ii), the critical exponent $\beta$ becomes 1 for $n=1$ [11-14], and this value is suggested to be universal irrespective of $n \in \mathbb{N}$ [12].

Apart from the all-to-all coupling, couplings represented by complex networks are of interest, like random graphs, scale-free networks, and small-world networks [18]. In particular, we focus on the small-world network because it is ubiquitous in the real world [19], and it is a notable network for the synchronization. The synchronization transition appears with the critical exponent $\beta=1 / 2$ in small-world networks even if they are very close to the one-dimensional lattice [20], while the one-dimensional lattice hardly shows synchronization [20-22]. The previous research [20], however, fails to consider universality since it has treated only case (i) with $n=1$, whereas other universality classes might 
be hidden in other cases as mentioned above. In this paper, we numerically study the synchronization transitions in smallworld networks in all the cases, (i), (ii), and (iii), with varying values of $n$. Our results suggest that the critical exponent is universally $\beta=1 / 2$ for any $n \in \mathbb{N}$ in cases (i) and (ii), where the transition is continuous, while discontinuity in case (iii) is inherited.

This paper is organized as follows. In Sec. II, we briefly introduce the small-world network and coupled phase-oscillator models on it. We also introduce a family of natural frequency distributions, whose second-leading term is of the order $2 n$. In Sec. III, we show the finite-size scaling to calculate the critical exponent $\beta$. A similarity between systems in the small-world network and noisy systems is discussed in Sec. IV. Finally, in Sec. V, we summarize this paper and note some future works.

\section{COUPLED PHASE-OSCILLATOR MODELS ON SMALL-WORLD NETWORKS}

A coupled phase-oscillator model is defined by

$$
\begin{aligned}
\frac{\mathrm{d} \theta_{i}}{\mathrm{~d} t} & =\omega_{i}+\frac{K}{2 k} \sum_{j \in \Lambda_{i}} f_{a}\left(\theta_{j}-\theta_{i}\right), \\
f_{a}(\theta) & =\sin \theta+a \sin 2 \theta,
\end{aligned}
$$

for $i=1, \ldots, N . \theta_{i}$ and $\omega_{i}$ are the phase and the natural frequency of the $i$ th oscillator, respectively, and $\omega_{i}$ is randomly drawn from a natural frequency distribution, $g(\omega) . K>0$ is a coupling constant, describing the strength of the coupling between oscillators. The index set $\Lambda_{i}$ contains the indexes of oscillators connecting to the $i$ th oscillator, and it determines the network of couplings. For instance, the all-to-all coupling gives $\Lambda_{i}=\{1, \ldots, N\}$, and the nearest-neighbor coupling on the one-dimensional lattice gives $\Lambda_{i}=\{i-1, i+1\}$.

The coupling network represented by $\left\{\Lambda_{i}\right\}_{i=1}^{N}$ is arbitrarily chosen. In this paper, we are interested in the small-world network, which possesses the properties of a small diameter and a large clustering coefficient despite its sparsity. The small-world network can be seen in various fields of the real world, such as human relationships, the World Wide Web, and citations of scientific papers. In 1998, Watts and Strogatz proposed a breakthrough network model showing the properties of a small-world network, which is created in the following algorithm [19]. We first make a periodic $k$-nearest-neighbor network with $N$ nodes, which results in $k N$ links. Then we rewire each link with probability $p$, keeping in mind that we do not allow self-loops or link duplications. Moreover, we use only connected small-world networks: if a generated network is disconnected, we discard it and generate another one until a connected one is created. See Fig. 1 for a comparison between the all-to-all network and a small-world network. In this paper, we use the Watts-Strogatz small-world network with $k=3$ and $p=0.2$, following the previous research [20] which shows emergence of the synchronization transition in a small-world network.

As the natural frequency distribution $g(\omega)$, we introduce a family of distributions parametrized by a natural number $n \in$ $\mathbb{N}$

$$
g_{n}(\omega)=\frac{n}{\Gamma(1 /(2 n)) \Delta} e^{-(\omega / \Delta)^{2 n}},
$$
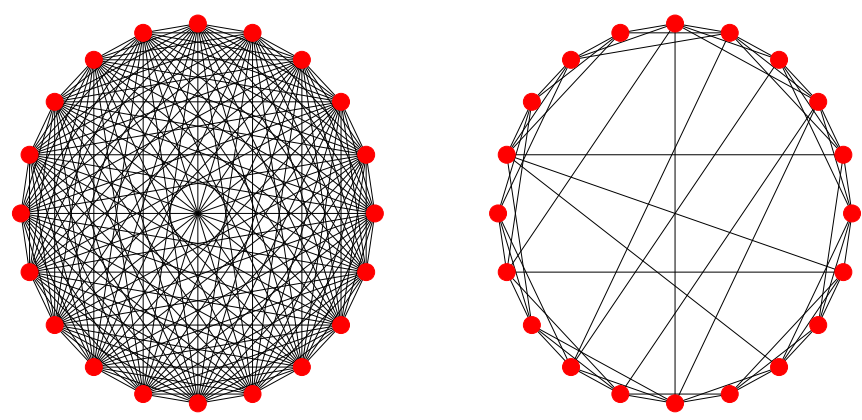

FIG. 1. Comparison between the all-to-all network (left) and a small-world network (right) with 20 nodes. The small-world network is constructed from the $k$-nearest-neighbor lattice $(k=3)$ with rewiring probability $p=0.2$.

where $\Gamma(z)=\int_{0}^{\infty} t^{z-1} e^{-t} \mathrm{~d} t$ is the Gamma function defined on $\operatorname{Re}(z)>0$. Here, $\Delta>0$ is a parameter describing the width of the distribution. We note that $n=1$ gives the Gaussian distribution. The distribution $g_{n}(\omega)$ is unimodal and symmetric with respect to $\omega=0$, and its Maclaurin expansion has the form

$$
g_{n}(\omega)=g_{n}(0)-C_{n} \omega^{2 n}+\cdots,
$$

where $C_{n}=n /\left(\Gamma(1 /(2 n)) \Delta^{2 n+1}\right)$ is positive. We remark that the generalized Lorentzian distribution introduced in Ref. [23] also has the same expansion form up to the second-leading term. In the limit $n \rightarrow \infty, g_{n}(\omega)$ converges to $g_{\infty}(\omega)$ in the $L^{1}$ norm:

$$
g_{\infty}(\omega)= \begin{cases}1 /(2 \Delta), & \omega \in(-\Delta, \Delta) \\ 0 & \text { otherwise. }\end{cases}
$$

This distribution is a uniform distribution on a compact support. See Fig. 2 for graphs of the distributions $g_{n}(\omega)$ and convergence to $g_{\infty}(\omega)$.

To visualize the extent of synchronization of oscillators, we introduce the order parameter $r_{N}$ defined by

$$
r_{N}=\left|\frac{1}{N} \sum_{j=1}^{N} e^{i \theta_{j}}\right| \text {. }
$$

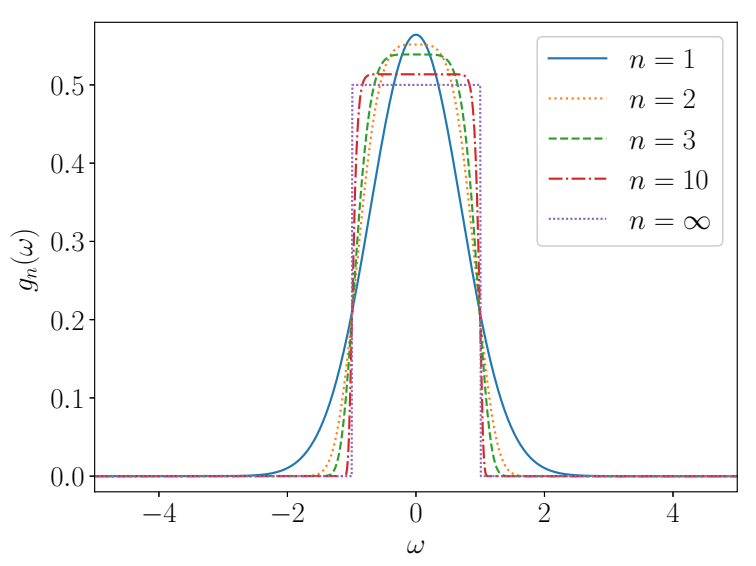

FIG. 2. Graphs of $g_{n}(\omega)$ with $n=1,2,3,10$, and $\infty$, where we set $\Delta=1$. $g_{n}(\omega)$ converges to $g_{\infty}(\omega)$ in the limit $n \rightarrow \infty$. 
The order parameter represents the centroid of the oscillators moving on the complex unit circle $\mathbb{S}^{1}$. When the oscillators are uniformly distributed on $\mathbb{S}^{1}$, which corresponds to the nonsynchronized state, $r_{N}$ gets close to 0 . On the other hand, when the oscillators gather at a point on $\mathbb{S}^{1}$, which corresponds to the synchronized state, $r_{N}$ equals 1 . The order parameter $r_{N}$ is therefore useful for monitoring synchronization of the coupled phase-oscillator models. In the next section, we look into the dependency of the order parameter $r_{N}$ on the coupling strength $K$.

The coupled phase-oscillator model of the small-world network represented by Eq. (1) has been considered previously $[24,25]$, but we stress that the numbers of links are completely different from ours. From the construction algorithm, a small-world network has $k N$ links and $k=3$ in our networks but $k=O(N)$ in the literature. An advantage of networks with $k=O(N)$ is that they can be analyzed through the equation of continuity [26]. Nevertheless, this advantage implies at the same time that such a small-world network in the literature is essentially the same as the all-to-all coupling and is not suitable for detecting new universality classes.

\section{NUMERICAL SIMULATIONS}

In the large population limit $N \rightarrow \infty$, the coupled phaseoscillator model, Eq. (1), is expected to show a synchronization transition around a critical point $K_{\mathrm{c}}$. For $K<K_{\mathrm{c}}$, the order parameter $r(K):=\lim _{N \rightarrow \infty} r_{N}(K)$ is 0 , which corresponds to the nonsynchronized state. On the other hand, for $K>K_{\mathrm{c}}$, the model shows partially synchronized states, in which $r(K)$ exhibits power-law behavior close to the critical point in the form of

$$
r(K) \sim\left(K-K_{\mathrm{c}}\right)^{\beta},
$$

where $\beta$ is one of the critical exponents. The critical exponents are crucial to describe critical phenomena, and models are classified into universality classes, each of which shares the same critical exponents. Calculating the critical exponents, including $\beta$, is therefore an important topic from theoretical and numerical perspectives.

\section{A. Finite-size scaling}

The critical exponent $\beta$ is defined in the large population limit $N \rightarrow \infty$, but the limit cannot be achieved through numerical simulations. To overcome this difficulty, we use the finite-size scaling theory, which provides us the limit from observations in finite-size systems. The first assumption of our finite-size scaling theory is the existence of the coherent number $N_{\mathrm{c}}(K)$ [27] diverging at the critical point $K=K_{\mathrm{c}}$ as

$$
N_{\mathrm{c}}(K) \propto\left(K-K_{\mathrm{c}}\right)^{-\bar{\nu}},
$$

where $\bar{v}$ is another unknown positive critical exponent. The coherent number corresponds to the correlation length in a simple lattice model. The second assumption is that the order parameter $r_{N}(K)$ depends on $K$ only through the ratio

$$
\frac{N}{N_{\mathrm{c}}(K)} \propto\left[\left(K-K_{\mathrm{c}}\right) N^{1 / \bar{v}}\right]^{\bar{\nu}} .
$$

These assumptions imply that $r_{N}(K)$ can be represented by

$$
r_{N}(K)=N^{-\beta / \bar{v}} F\left(\left(K-K_{\mathrm{c}}\right) N^{1 / \bar{v}}\right),
$$

where the function $F$, which is called the scaling function, must be

$$
F(x) \propto x^{\beta} \quad \text { for large } \quad x
$$

to reproduce the critical exponent $\beta$ in the limit $N \rightarrow \infty$. We remark that the exponent $\beta / \bar{v}$ expresses the finite-size fluctuation of $r_{N}(K)$ at the critical point $K=K_{\text {c }}$.

The finite-size scaling is widely used for numerical studies of critical phenomena in continuous phase transitions, including coupled phase-oscillator models [20,28-33]. An important remark on Eq. (9) is that, on the $\left(\left(K-K_{\mathrm{c}}\right) N^{1 / \bar{v}}, N^{\beta / \bar{v}} r_{N}\right)$ plane, observed values of $r_{N}(K)$ must collapse on a single graph of $F$ for any values of $N$ and $K$. The unknown values of $K_{\mathrm{c}}, \beta$, and $\bar{v}$ are determined by detecting the best-fit values. The detection is performed by using the Bayesian scaling analysis $[34,35]$, for which a brief introduction is given in the Appendix.

\section{B. Computation of the order parameter}

We determine the value of the order parameter $r_{N}(K)$ for a given set of $(N, K)$ through temporal evolution of the system and two steps of averaging. The model equation, Eq. (1), is numerically integrated by using the fourth-order Runge-Kutta algorithm with the time step $\delta t=0.1$. Initial values of the phases $\left\{\theta_{i}\right\}$ are randomly drawn from the uniform distribution on the interval $[0,2 \pi)$, and the natural frequencies $\left\{\omega_{i}\right\}$ are randomly drawn from the distribution function $g_{n}(\omega)$. The order parameter $r_{N}$ defined by Eq. (5) depends on time $t$, and we take the time average in the time interval $t \in[300,500]$. This is the first averaging.

Further, we perform 400 realizations by changing smallworld networks, the initial values of $\left\{\theta_{i}\right\}$, and $\left\{\omega_{i}\right\}$ for a given set of $(N, K)$. To compute the confidence interval of the order parameter, the resampling technique is in use. We choose 200 samples of 400 realizations and calculate the mean of the time-averaged order parameter in the chosen 200 samples. The mean of the $i$ th resampling is denoted $r_{N}^{(i)}(K)$, and we perform the resampling for $S=1000$ times. The value $r_{N}(K)$ is determined by taking the second averaging over $S$ samples $\left\{r_{N}^{(i)}(K)\right\}_{i=1}^{S}$, which also provide the confidence interval of $r_{N}(K)$.

See Fig. 3 for the obtained $r_{N}(K)$ for $a=0$ and -0.2 with $n=1$, where the condition $a \leqslant 0$ is expected to give a continuous transition. In the following two sections, we compute the critical exponents for $a=0$ and $a=-0.2$ and show discontinuity for $a=0.5$, respectively. We remark that $a=-0.2$ and 0.5 are not special values. They are arbitrarily chosen from a neighborhood of $a=0$ to demonstrate differences among the three cases of (i) $a=0$, (ii) $a<0$, and (iii) $a>0$.

\section{Critical exponents for continuous transition}

The finite-size scaling, Eq. (9), is a powerful tool to compute the unknown values of $K_{\mathrm{c}}, \beta$, and $\bar{\nu}$, but it is not perfect if $N$ is not sufficiently large. We thus compute the unknown 


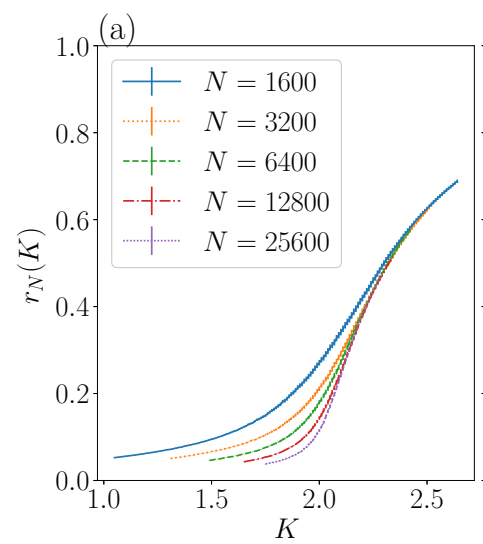

(b)

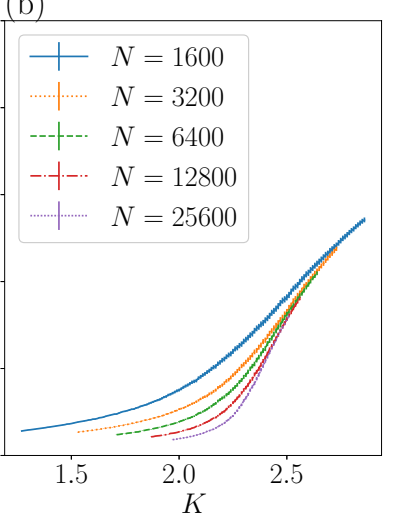

FIG. 3. Graphs of order parameter $r_{N}(K)$ with its confidence interval for the model, (1), where we take the coupling function $f_{a}(\theta)$ with (a) $a=0$ and (b) $a=-0.2$. As a natural frequency distribution, we use $g_{1}(\omega)$ with $\Delta=1$ and $N=1600,3200,6400,12800$, and 25600 , from top to bottom. $r_{N}(K)$ and its confidence interval are evaluated by the resampling technique. Error bars are so small that they may not be visible.

variables for three values of $N \in\left\{N_{\min }, 2 N_{\min }, 4 N_{\min }\right\}$ and observe convergence by varying $N_{\min }$. Moreover, we use the resampling technique again to estimate the unknown values with their confidence intervals. Consequently, we have $S=$ 1000 sets of the three values for a given $N_{\min }$ as reported in Fig. 4 because each resampling set $r_{N}^{(i)}(K)$ determines them. Finally, the values and the confidence intervals of $K_{\mathrm{c}}, \beta$, and $\bar{v}$ are computed as the averages and the standard deviations over $S=1000$ sets. The estimated values are verified in Fig. 5, where all the points lie on a single curve representing the scaling function $F$ for $N_{\min }=6400$.

The estimated values of $K_{\mathrm{c}}, \beta$, and $\bar{v}$ are summarized in Table I. The row of $N_{\min }=\infty$ is obtained by extrapolation from $N_{\min }=1600,3200$, and 6400 as demonstrated in Fig. 6 . We note that the extrapolated values of $\beta$ are close to $1 / 2$ and those of $\bar{v}$ are close to $5 / 2$ irrespective of the values of $a$ and $n$. The universality is completely unlike the all-to-all interaction case. Here we note that this result shares the same

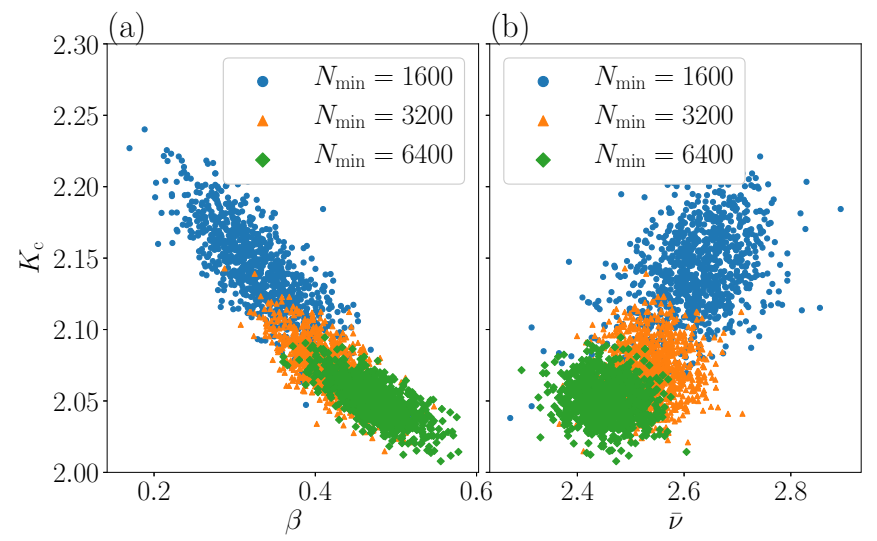

FIG. 4. Scattering plots of computed parameters (a) $\left(\beta, K_{\mathrm{c}}\right)$ and (b) $\left(\bar{v}, K_{\mathrm{c}}\right)$, evaluated by the Bayesian scaling analysis. Here, we use $(a, n)=(0,1)$, and we set $N_{\min }$ to 1600,3200 , and 6400 .

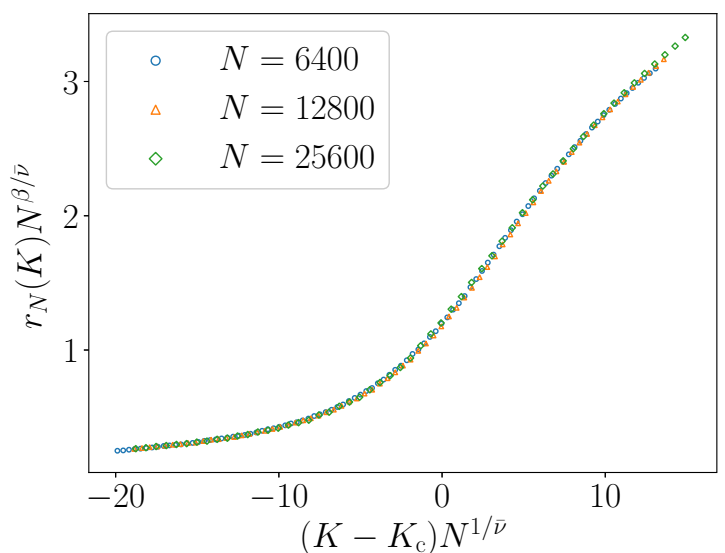

FIG. 5. Graph of scaled order parameter $r_{N}(K) N^{\beta / \bar{v}}$ versus scaled coupling constant $\left(K-K_{\mathrm{c}}\right) N^{1 / \bar{v}}$ for $(a, n)=(0,1)$, where we use $\beta, \bar{v}$, and $K_{\mathrm{c}}$, obtained by the Bayesian scaling analysis for $N_{\min }=6400$. The values of $\beta, \bar{v}$, and $K_{\mathrm{c}}$ are listed in Table I. We see that the scaled data are well collapsed to the scaling function $F$.

critical exponent $\bar{v}=5 / 2$ as the all-to-all interaction case for $(a, n)=(0,1)$ calculated in Ref. [36].

The value $\bar{v} \simeq 5 / 2$ is not in agreement with the value $\bar{v} \simeq$ 2 previously reported for $(a, n)=(0,1)$ [20]. We suppose that the discrepancy comes from the method to compute the critical exponents. In the literature, the authors used the fact that $r_{N}(K) N^{\beta / \bar{v}}$ takes a constant value irrespective of $N$ at the critical point $K=K_{\mathrm{c}}$ [see Eq. (9)]. Using this fact, they first find the best-fit values of $\beta / \bar{\nu}$ and $K_{\mathrm{c}}$ by varying the system size $N$. One more equation is obtained by derivating the finite-size scaling, Eq. (9), which produces

$$
\log \left[\frac{\mathrm{d} r_{N}}{\mathrm{~d} K}\left(K_{\mathrm{c}}\right)\right]=\frac{1-\beta}{\bar{v}} \log N+\text { const. }
$$

Plotting the left-hand side as a function of $\log N$, one has the slope $(1-\beta) / \bar{\nu}$. A remarkable disadvantage of this method is that the estimation relies on a high precision of $r_{N}(K)$ around the critical point $K=K_{\mathrm{c}}$, while the Bayesian scaling analysis uses $r_{N}(K)$ in a wider interval of $\left(K-K_{\mathrm{c}}\right) N^{1 / \bar{v}}$ and provides persistence against fluctuation. We, therefore, believe that $\bar{v} \simeq$ $5 / 2$ obtained by the Bayesian scaling analysis is more reliable.

\section{Discontinuity of transition}

In the all-to-all interaction a positive $a$ induces discontinuity of the synchronization transition [12]. We reveal that the transition is discontinuous also in a small-world network. The discontinuity appears as a result of a subcritical transition, and a subcritical transition has metastability: A partially synchronized state is stable in addition to a stable nonsynchronized state for a fixed $K$ close to the critical point. The metastability implies that the final state depends on the choice of the initial state, and the dependency is extracted by observing hysteresis.

Fixing $a=0.5$, we check the existence of hysteresis by preparing two sets of the initial phases $\left\{\theta_{i}\right\}_{i=1}^{N}$ for each $K$ : (i) We start from $K=K_{\text {start }}$, where $K_{\text {start }}$ is sufficiently smaller than the critical value $K_{\mathrm{c}}$, and the initial phases $\left\{\theta_{i}\right\}_{i=1}^{N}$ are 
TABLE I. Critical exponents $\beta$ and $\bar{v}$ and the critical point $K_{\mathrm{c}}$ of (1) depending on the coupling function $f_{a}(\theta)=\sin \theta+a \sin 2 \theta$ and the natural frequency distribution $g_{n}(\omega)$ in Eq. (2), for $a=0$ and -0.2 and $n=1,2,3$, and $\infty$. For each pair of $(a, n)$, we use $N_{\min }=1600,3200$, and 6400 and execute the Bayesian scaling analysis [34] to find the best parameter fitting, (9). We extrapolate the critical values to $N_{\text {min }}=\infty$ by using the least squares method, and they are listed on the row $N_{\min }=\infty$. Here, we show the confidence intervals for the last digit of the estimated values in parentheses; for example, 2.13(3) $=2.13 \pm 0.03$.

\begin{tabular}{|c|c|c|c|c|c|}
\hline$f_{a}(\theta)$ & $g_{n}(\omega)$ & $N_{\min }$ & $K_{\mathrm{c}}$ & $\beta$ & $\bar{v}$ \\
\hline \multirow[t]{16}{*}{$a=0$} & \multirow[t]{4}{*}{$n=1$} & 1600 & $2.13(3)$ & $0.33(4)$ & $2.61(7)$ \\
\hline & & 3200 & $2.07(1)$ & $0.42(3)$ & $2.53(5)$ \\
\hline & & 6400 & $2.05(1)$ & $0.47(3)$ & $2.45(4)$ \\
\hline & & $\infty$ & $2.02(2)$ & $0.51(4)$ & $2.40(6)$ \\
\hline & \multirow[t]{4}{*}{$n=2$} & 1600 & $1.85(1)$ & $0.27(2)$ & $2.67(5)$ \\
\hline & & 3200 & $1.78(1)$ & $0.37(2)$ & $2.53(3)$ \\
\hline & & 6400 & $1.755(9)$ & $0.44(2)$ & $2.50(3)$ \\
\hline & & $\infty$ & $1.72(1)$ & $0.49(2)$ & $2.43(4)$ \\
\hline & \multirow[t]{4}{*}{$n=3$} & 1600 & $1.80(1)$ & $0.28(2)$ & $2.62(4)$ \\
\hline & & 3200 & $1.76(1)$ & $0.33(2)$ & $2.51(3)$ \\
\hline & & 6400 & $1.723(8)$ & $0.44(2)$ & $2.51(3)$ \\
\hline & & $\infty$ & $1.69(1)$ & $0.47(2)$ & $2.46(4)$ \\
\hline & \multirow[t]{4}{*}{$n=\infty$} & 1600 & $1.83(1)$ & $0.27(1)$ & $2.50(4)$ \\
\hline & & 3200 & $1.79(1)$ & $0.36(2)$ & $2.52(3)$ \\
\hline & & 6400 & $1.780(8)$ & $0.41(2)$ & $2.46(3)$ \\
\hline & & $\infty$ & $1.76(1)$ & $0.46(2)$ & $2.46(4)$ \\
\hline \multirow[t]{16}{*}{$a=-0.2$} & \multirow[t]{4}{*}{$n=1$} & 1600 & $2.43(5)$ & $0.38(8)$ & $2.67(9)$ \\
\hline & & 3200 & $2.35(2)$ & $0.44(6)$ & $2.58(7)$ \\
\hline & & 6400 & $2.34(1)$ & $0.45(4)$ & $2.42(6)$ \\
\hline & & $\infty$ & $2.31(3)$ & $0.48(6)$ & $2.36(8)$ \\
\hline & \multirow[t]{4}{*}{$n=2$} & 1600 & $2.09(3)$ & $0.31(4)$ & $2.87(7)$ \\
\hline & & 3200 & $1.99(2)$ & $0.41(4)$ & $2.65(5)$ \\
\hline & & 6400 & $1.96(1)$ & $0.47(3)$ & $2.52(4)$ \\
\hline & & $\infty$ & $1.91(2)$ & $0.51(4)$ & $2.41(6)$ \\
\hline & \multirow[t]{4}{*}{$n=3$} & 1600 & $2.04(3)$ & $0.27(5)$ & $2.85(8)$ \\
\hline & & 3200 & $1.96(2)$ & $0.37(4)$ & $2.65(5)$ \\
\hline & & 6400 & 1.91(1) & $0.49(3)$ & $2.59(4)$ \\
\hline & & $\infty$ & $1.86(2)$ & $0.55(4)$ & $2.50(6)$ \\
\hline & \multirow{4}{*}{$n=\infty$} & 1600 & $2.08(3)$ & $0.25(4)$ & $2.76(6)$ \\
\hline & & 3200 & $2.00(1)$ & $0.38(4)$ & $2.69(5)$ \\
\hline & & 6400 & $1.97(1)$ & $0.43(3)$ & $2.54(4)$ \\
\hline & & $\infty$ & $1.94(2)$ & $0.49(4)$ & $2.49(6)$ \\
\hline
\end{tabular}

randomly drawn from the interval $[0,2 \pi)$. At a certain value of $K$, the final phases at $t=500$ are used as the initial phases at the successive value $K+\Delta K$ in the increasing direction. The increase in $K$ is continued up to $K=K_{\text {end }}$, where $K_{\text {end }}$ is sufficiently larger than the critical value $K_{\mathrm{c}}$. We call process (i) the "forward" process, and $r_{N}^{\text {(forward) }}(K)$ denotes its order parameter. (ii) Contrary to the forward process, we start with the random initial phases $\left\{\theta_{i}\right\}_{i=1}^{N}$ at $K=K_{\text {end }}$ and decrease $K$ up to $K=K_{\text {start }}$ following the same procedure as the forward process. We call this process the "backward" process, and $r_{N}^{\text {(backward) }}(K)$ denotes its order parameter. We have executed the numerical simulations of Eq. (1) for $a=0,-0.2$, and 0.5, and $n=1,2,3$, and $\infty$. For the system size $N=25600$, the hysteresis appears only for $a=0.5$ regardless of $n$ as illustrated in Fig. 7 for $n=1$. We have checked that $t=500$ is sufficiently long to pass the transient period, and simulations up to $t=800$ do not affect the hysteresis. We therefore conclude that the system represented by Eq. (1) shows a discontinuous transition for $a=0.5$ as the all-to-all interaction case.

\section{SMALL-WORLD NETWORK AND NOISE}

We discuss similarity between systems in small-world networks and noise systems. For simplicity, we consider the Kuramoto model $(a=0)$ briefly. The steady state in the Kuramoto model is proportional to $\delta(\omega-K r \sin \theta)$ in the synchronized regime of $\omega$ [8,37], where $\delta$ is Dirac's delta function. The $\delta$ function with the integration over $\omega$ and symmetry of the natural frequency distribution yield the selfconsistent equation of the order parameter $r$ as

$$
r=K r \int_{-\pi / 2}^{\pi / 2} g_{n}(K r \sin \theta) \cos ^{2} \theta \mathrm{d} \theta .
$$

The order parameter $r$ is sufficiently small around the critical point and we perform the Maclaurin expansion of $g_{n}$. The leading order of the expansion, which is $O(r)$, determines the celebrated critical point $K_{\mathrm{c}}=2 /\left[\pi g_{n}(0)\right]$. The partially synchronized branch is obtained by balancing the secondleading order of $O\left(r^{2 n+1}\right)$ with the first-leading order of 


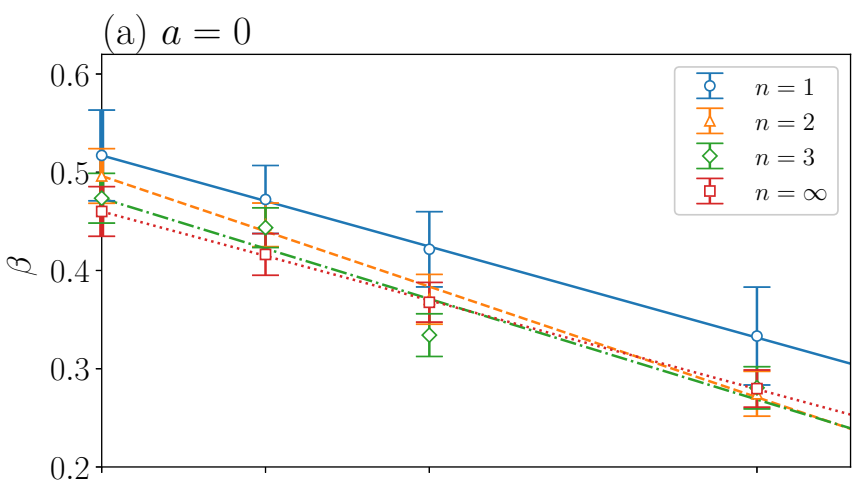

(b) $a=-0.2$

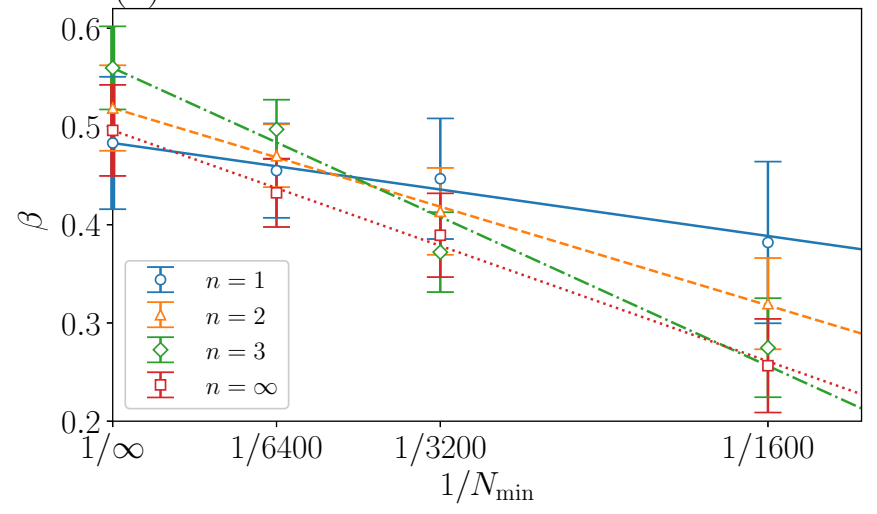

FIG. 6. Graphs of $\beta$ as a function of $1 / N_{\min }$ for (a) $a=0$ and (b) $a=-0.2$ in Eq. (1). Critical exponents obtained by the finitesize scaling are shown with error bars, and the least squares method shows the extrapolations at the left boundary of the panels. For each $a$, the resulting linear regression lines are drawn with the solid line for $n=1$, the dashed line for $n=2$, the dot-dashed line for $n=3$, and the dotted line for $n=\infty$.

$O\left(r\left(K-K_{\mathrm{c}}\right)\right)$, and the balance results as $r \propto\left(K-K_{\mathrm{c}}\right)^{1 /(2 n)}$. We then obtain the critical exponent $\beta=1 /(2 n)$.

On the contrary, in a small-world network, a steady state is not written in the form of the $\delta$ function and the synchronized oscillators are still "noisy" as shown in Fig. 8. The synchronized oscillators no longer capture the flatness of $g_{n}(\omega)$ around $\omega=0$, and the critical exponent $\beta$ falls into the classical value $1 / 2$ regardless of the natural frequency distribution $g_{n}(\omega)$ as a noisy system [38].

Moreover, in the model having the nonvanishing second harmonics of the coupling function with $a<0$, the noise recovers $\beta=1 / 2$ [11], whereas the no-noise system gives $\beta=1$ [39]. The universality of $\beta=1 / 2$ observed in systems in small-world networks is therefore very similar to that in noisy systems.

\section{DISCUSSION AND CONCLUSION}

We calculated the critical exponents $\beta$ and $\bar{v}$ for coupled phase-oscillator models on small-world networks by using the finite-size scaling method. We set the coupling function as $f_{a}(\theta)=\sin \theta+a \sin 2 \theta$ and the natural frequency distribution as $g_{n}(\omega)$ defined in Eq. (2), and we studied the $(a, n)$ dependency of the critical exponents. Our numerical results
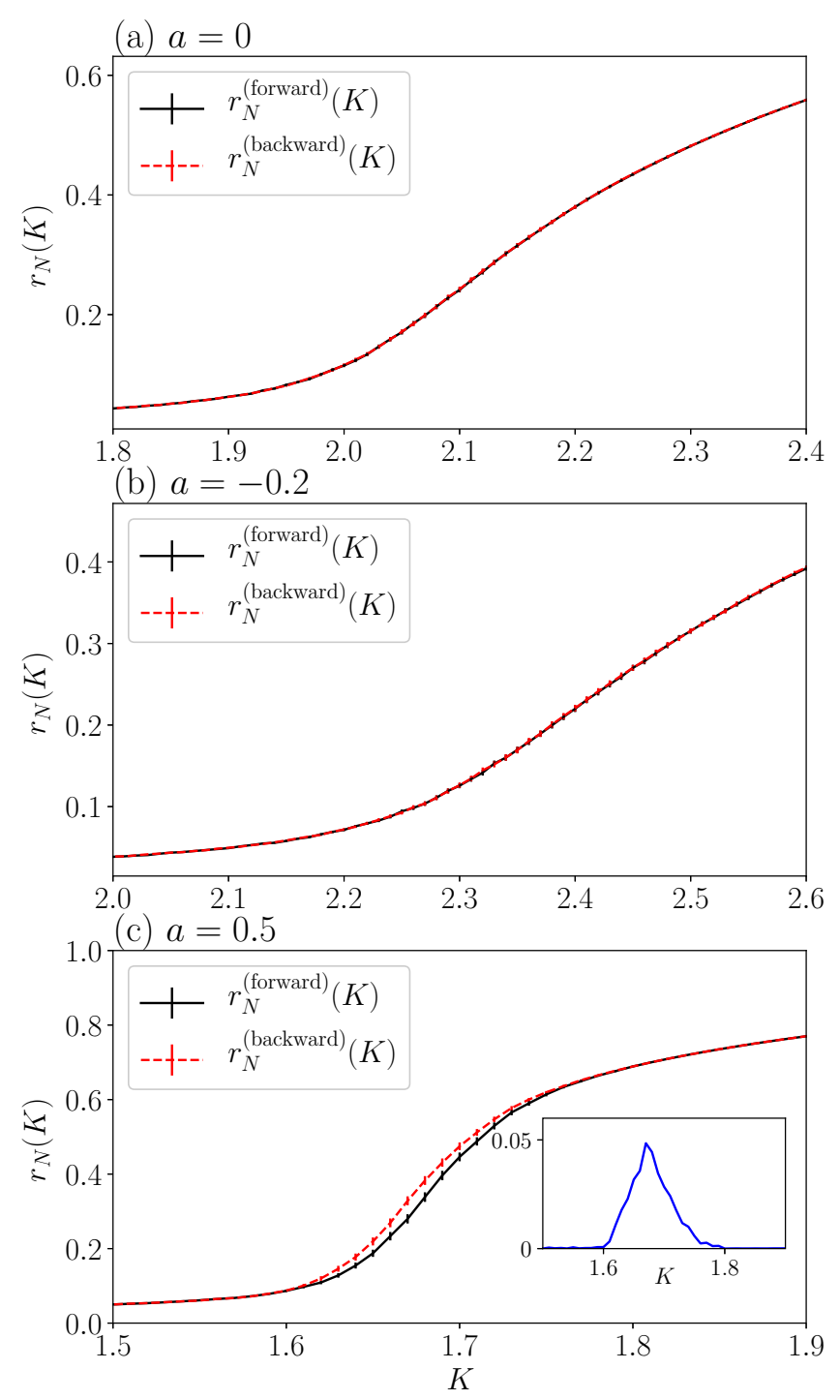

FIG. 7. Graphs of $r_{N}(K)$ and its error bar of (1) for (a) $(a, n)=$ $(0,1)$, (b) $(a, n)=(-0.2,1)$, and (c) $(a, n)=(0.5,1)$ with two different types of initial phases, where we set the number of oscillators $N=25600$. We see that, only in (c), $r_{N}(K)$ takes a different value depending on the choice of the initial phases around $K \in(1.6,1.8)$. Inset in (c): Graph of $r_{N}^{\text {(backward) }}(K)-r_{N}^{\text {(forward) }}(K)$.

suggest that $\beta=1 / 2$ and $\bar{v}=5 / 2$ for all $g_{n}(\omega)$ and coupling function $f_{a}(\theta)$ with $a=0$ and -0.2 . This universality shows a sharp contrast with the all-to-all interaction case, which has various values of $\beta$ depending on the coupling function and the natural frequency distribution. A possible explanation of the source of contrast can be found in the number of links of networks considered: our small-world network has $O(N)$ links, while the all-to-all interaction has $O\left(N^{2}\right)$ links. We have also found that the model, Eq. (1), shows a discontinuous transition for $a=0.5$. The (dis)continuity is a weaker property than the values of the critical exponents, and it is shared between the two types of networks: networks with $O(N)$ links and networks with $O\left(N^{2}\right)$ links.

We end this paper by commenting on two future works. First, we picked up two representative points of $a$ from a 

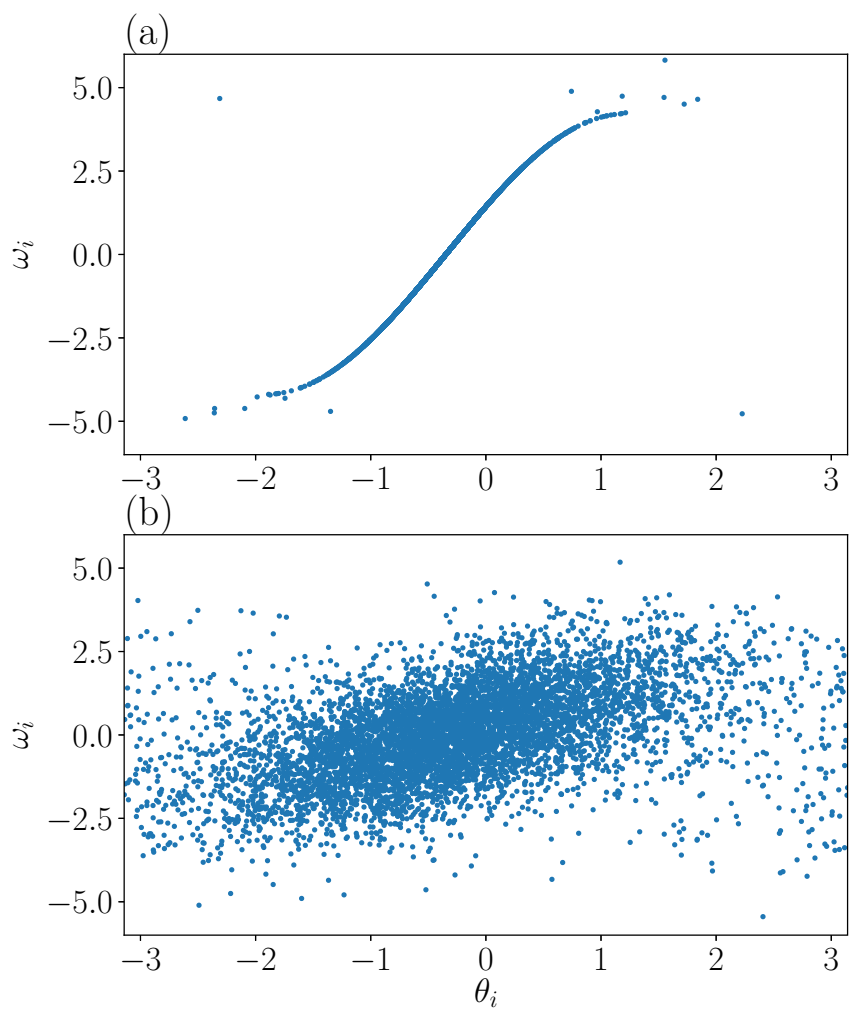

FIG. 8. Snapshots of oscillators on the $\left(\theta_{i}, \omega_{i}\right)$ plane at $t=500$. (a) The all-to-all network. (b) A small-world network. The system size $N=6400$. The coupling constant $K=4.5$. $(a, n)=(0,1)$.

neighborhood of $a=0$ to investigate universality of the critical exponents. Studying a global phase diagram on the $(K, a)$ plane is a subject for future research. Second, we note the universal value $\beta=1 / 2$ in the Kuramoto model, which is recovered by adding noise regardless of the natural frequency distribution [38]. A small-world network may play a role of noise due to inhomogeneous couplings, and another project to do is to make a bridge between a noisy Kuramoto model and a model on a small-world network.

\section{ACKNOWLEDGMENTS}

In this research work we used the supercomputer of ACCMS, Kyoto University. R.Y. acknowledges the support of an Iwadare Scholarship from the Iwadare Scholarship Foundation. Y.Y.Y. acknowledges the support of JSPS KAKENHI Grant No. 16K05472.

\section{APPENDIX: BAYESIAN SCALING ANALYSIS}

We briefly review the Bayesian scaling analysis [34,35], a statistical method for estimating the values such as $\beta$, $\bar{v}$, and
$K_{\mathrm{c}}$ in Eq. (9). We write these values as $\theta_{p}=\left(\beta, \bar{v}, K_{\mathrm{c}}\right)$. We assume that the scaling function $F$ in Eq. (9) obeys a Gaussian process,

$$
F \sim \mathcal{G P}\left(m, k_{\theta_{h}}\right),
$$

with mean function $m(\cdot)$ and covariance kernel $k_{\theta_{h}}(\cdot, \cdot)$. Here $\theta_{h}$ denotes the hyperparameters of the covariance kernel. We also set $m=0$ for simplicity. In the following, we also use the notation $\boldsymbol{\theta}=\left(\theta_{h}, \theta_{p}\right)$. For the data $\left\{r_{N_{i}}\left(K_{i}\right)\right\}_{i=1}^{M}$, the rescaled data $X_{\theta_{p}, i}=\left(K_{i}-K_{\mathrm{c}}\right) N_{i}^{1 / \bar{v}}$ and $Y_{\theta_{p}, i}=r_{N_{i}}\left(K_{i}\right) N_{i}^{\beta / \bar{v}}$ must collapse on the scaling function as $Y_{\theta_{p}, i}=F\left(X_{\theta_{p}, i}\right)$. Since $F$ is a Gaussian process, $Y_{\theta_{p}}$ obeys an $M$-dimensional Gaussian distribution, and the probability of $Y$ for the parameter $\boldsymbol{\theta}$ is

$$
\begin{aligned}
p(Y \mid \boldsymbol{\theta}) & =\mathcal{N}\left(Y_{\theta_{p}} \mid \mathbf{0}, K_{\theta}\right) \\
& =\frac{1}{(2 \pi)^{N / 2}\left[\operatorname{det} K_{\theta}\right]^{1 / 2}} \exp \left[-\frac{1}{2} Y_{\theta_{p}}^{\top} K_{\theta}^{-1} Y_{\theta_{p}}\right] .
\end{aligned}
$$

Here, $\left[K_{\theta}\right]_{i, j}=k_{\theta_{h}}\left(X_{\theta_{p}, i}, X_{\theta_{p}, j}\right)$ is an $M \times M$-dimensional matrix. By assuming that the prior distribution of $\theta$ is uniform, we have

$$
p(\boldsymbol{\theta} \mid Y) \propto p(Y \mid \boldsymbol{\theta})
$$

from Bayes' theorem. The most probable parameters $\boldsymbol{\theta}$ are, therefore, estimated by finding the minimum of the likelihood function given by

$$
L_{\theta}=\log \left(\operatorname{det} K_{\theta}\right)+Y_{\theta_{p}}^{\top} K_{\theta}^{-1} Y_{\theta_{p}},
$$

which is obtained by taking the log and discarding the constants in Eq. (A2). The gradient of $L_{\boldsymbol{\theta}}$ for an element $\theta \in \boldsymbol{\theta}$ is given by

$\frac{\partial L_{\theta}}{\partial \theta}=\operatorname{tr}\left[K_{\theta}^{-1} \frac{\partial K_{\theta}}{\partial \theta}\right]-\left(K_{\theta}^{-1} Y_{\theta_{p}}\right)^{\top} \frac{\partial K_{\theta}}{\partial \theta}\left(K_{\theta}^{-1} Y_{\theta_{p}}\right)+2 Y_{\theta_{p}}^{\top} K_{\theta}^{-1} \frac{\partial Y_{\theta_{p}}}{\partial \theta}$,

and using this gradient, the gradient method gives us the most probable parameters $\boldsymbol{\theta}$.

In this paper, we consider a kernel based on a radial basis function kernel,

$$
k_{\theta_{h}}(x, y)=\theta_{1} \exp \left[-\frac{(x-y)^{2}}{\theta_{2}}\right]+\theta_{3} \delta(x, y),
$$

which is parameterized by $\theta_{h}=\left(\theta_{1}, \theta_{2}, \theta_{3}\right)$ with $\theta_{1,2,3}>0$, and $\delta(x, y)=1$ when $x=y$; otherwise, $\delta(x, y)=0$. Here, $\theta_{3}$ denotes the data fidelity. Roughly speaking, a sample path of the Gaussian process associated with a radial basis function kernel are known to be an infinitely differentiable function; see [40, corollary 4.13] for a rigorous statement. Therefore, the Bayesian scaling analysis only assumes the smoothness of a scaling function, and it does not need an explicit form. See Refs. [34,35] for more detailed discussions.
[1] I. Aihara, T. Mizumoto, T. Otsuka, H. Awano, K. Nagira, H. G. Okuno, and K. Aihara, Spatio-temporal dynamics in collective frog choruses examined by mathematical modeling and field observations, Sci. Rep. 4, 3891 (2014).
[2] H. M. Smith, Synchronous flashing of fireflies, Science 82, 151 (1935).

[3] J. Buck and E. Buck, Mechanism of rhythmic synchronous flashing of fireflies, Science 159, 1319 (1968). 
[4] J. Pantaleone, Synchronization of metronomes, Am. J. Phys. 70 992 (2002).

[5] A. T. Winfree, Biological rhythms and the behavior of populations of coupled oscillators, J. Theoret. Biol. 16, 15 (1967).

[6] Y. Kuramoto and H. Nakao, On the concept of dynamical reduction: The case of coupled oscillators, Phil. Trans. R. Soc. A 377, 20190041 (2019).

[7] Y. Kuramoto, Self-entertainment of a population of coupled non-linear oscillators, in International Symposium on Mathematical Problems in Theoretical Physics, edited by H. Araki, Lecture Notes in Physics Vol. 39 (Springer, Berlin, Heidelberg, 1975).

[8] S. H. Strogatz, From Kuramoto to Crawford: Exploring the onset of synchronization in populations of coupled oscillators, Physica D 143, 1 (2000).

[9] H. Chiba, A proof of the Kuramoto conjecture for a bifurcation structure of the infinite-dimensional Kuramoto model, Ergod. Theor. Dynam. Syst. 35, 762 (2015).

[10] H. Daido, Susceptibility of large populations of coupled oscillators, Phys. Rev. E 91, 012925 (2015).

[11] J. D. Crawford, Scaling and Singularities in the Entrainment of Globally Coupled Oscillators, Phys. Rev. Lett. 74, 4341 (1995).

[12] H. Chiba and I. Nishikawa, Center manifold reduction for large populations of globally coupled phase oscillators, Chaos $\mathbf{2 1}$, 043103 (2011).

[13] M. Komarov and A. Pikovsky, Multiplicity of Singular Synchronous States in the Kuramoto Model of Coupled Oscillators, Phys. Rev. Lett. 111, 204101 (2013).

[14] M. Komarov and A. Pikovsky, The Kuramoto model of coupled oscillators with a bi-harmonic coupling function, Physica D 289, 18 (2014).

[15] L. Basnarkov and V. Urumov, Phase transitions in the Kuramoto model, Phys. Rev. E 76, 057201 (2007).

[16] D. Pazó, Thermodynamic limit of the first-order phase transition in the Kuramoto model, Phys. Rev. E 72, 046211 (2005).

[17] H. Daido, Intrinsic fluctuations and a phase transition in a class of large populations of interacting oscillators, J. Stat. Phys. 60, 753 (1990).

[18] S. N. Dorogovtsev, A. V. Goltsev, and J. F. F. Mendes, Critical phenomena in complex networks, Rev. Mod. Phys. 80, 1275 (2008).

[19] D. J. Watts and S. H. Strogatz, Collective dynamics of 'smallworld' networks, Nature 393, 440 (1998).

[20] H. Hong, M. Y. Choi, and B. J. Kim, Synchronization on smallworld networks, Phys. Rev. E 65, 026139 (2002).

[21] H. Sakaguchi, S. Shinomoto, and Y. Kuramoto, Local and global self-entrainments in oscillator lattices, Prog. Theor. Phys. 77, 1005 (1987).
[22] H. Daido, Lower Critical Dimension for Populations of Oscillators with Randomly Distributed Frequencies: A Renormalization-Group Analysis, Phys. Rev. Lett. 61, 231 (1988).

[23] B. Pietras, N. Deschle, and A. Daffertshofer, First-order phase transitions in the Kuramoto model with compact bimodal frequency distributions, Phys. Rev. E 98, 062219 (2018).

[24] H. Chiba, G. S. Medvedev, and M. S. Mizuhara, Bifurcations in the Kuramoto model on graphs, Chaos 28, 073109 (2018).

[25] G. S. Medvedev, Small-world networks of Kuramoto oscillators, Physica D 266, 13 (2014).

[26] L. Lovász, Large Networks and Graph Limits (American Mathematical Society, Providence, RI, 2012).

[27] R. Botet, R. Jullien, and P. Pfeuty, Size Scaling for Infinitely Coordinated Systems, Phys. Rev. Lett. 49, 478 (1982).

[28] A. Pelisseto and E. Vicari, Critical phenomena and renormalization-group theory, Phys. Rep. 368, 549 (2002).

[29] M. Hasenbusch, Finite size scaling study of lattice models in the three-dimensional Ising universality class, Phys. Rev. B 82, 174433 (2010).

[30] A. Pikovsky and M. Rosenblum, Dynamics of globally coupled oscillators: Progress and perspectives, Chaos 25, 097616 (2015).

[31] H. Hong, H. Chaté, L-H. Tang, and H. Park, Finite-size scaling, dynamic fluctuations, and hyperscaling relation in the $\mathrm{Ku}-$ ramoto model, Phys. Rev. E 92, 022122 (2015).

[32] T. Coletta, R. Delabays, and P. Jacquod, Finite-size scaling in the Kuramoto model, Phys. Rev. E 95, 042207 (2017).

[33] R. Juhász, J. Kelling, and G. Ódor, Critical dynamics of the Kuramoto model on sparse random networks, J. Stat. Mech. (2019) 053403.

[34] K. Harada, Bayesian inference in the scaling analysis of critical phenomena, Phys. Rev. E 84, 056704 (2011).

[35] K. Harada, Kernel method for corrections to scaling, Phys. Rev. E 92, 012106 (2015).

[36] H. Hong, H. Chaté, H. Park, and L-H. Tang, Entrainment Transition in Populations of Random Frequency Oscillators, Phys. Rev. Lett. 99, 184101 (2007).

[37] J. D. da Fonseca and C. V. Abud, The Kuramoto model revisited, J. Stat. Mech. (2018) 103204.

[38] H. Sakaguchi, Cooperative phenomena in coupled oscillator systems under external fields, Prog. Theor. Phys. 79, 39 (1988).

[39] H. Daido, Generic Scaling at the Onset of Macroscopic Mutual Entrainment in Limit-Cycle Oscillators with Uniform All-ToAll Coupling, Phys. Rev. Lett. 73, 760 (1994).

[40] M. Kanagawa, P. Hennig, D. Sejdinovic, and B. K. Sriperumbudur, Gaussian processes and kernel methods: A review on connections and equivalences, arXiv:1807.02582. 